\section{Schwere Zwischenfälle sind selten}

\section{Über Inzidenz und klinische Charakteristika schwerer Zwischenfälle im Rahmen einer spezifischen Immuntherapie (SIT) gab es bisher wenig systematische Daten. Konkrete Hinweise liefert nun eine retrospektive Umfrage bei US-amerikanischen Allergologen.}

\begin{abstract}
A lle Mitglieder der American Academy of Allergy, Asthma and Immunology (AAAAI) erhielten einen Kurzfragebogen zu „fast tödlichen“ Zwischenfällen bei SIT-Injektionen. Fast tödlich war definiert als das Auftreten einer schweren Ateminsuffizienz und/oder Hypotonie, die eine Notfallbehandlung mit Adrenalin erforderlich gemacht hatte. Von 646 Antwortenden (27\% der Angeschriebenen) berichteten 273 über einen solchen Zwischenfall. Sie erhielten daraufhin einen detaillierteren, 105 Punkte umfassenden Fragebogen. 68 Rücklaufbögen konnten schließlich ausgewertet werden.

Für die Zeitspanne von 1990 bis 2001 wird die Inzidenz ungesicherter fast tödlicher Zwischenfälle im Beobachtungsgebiet USA und Kanada auf 23 pro Jahr, entsprechend 5,4 Ereignisse auf eine Million Injektionen, geschätzt. Die 68 per Fragebogen detailliert darge-
\end{abstract}

stellten Fälle wurden als gesicherte fast tödliche Ereignisse gewertet, was einer Inzidenz von 4,7 pro Jahr bzw. einer Reaktion auf eine Million Injektionen entspricht. Bei 46\% dieser Reaktionen litt

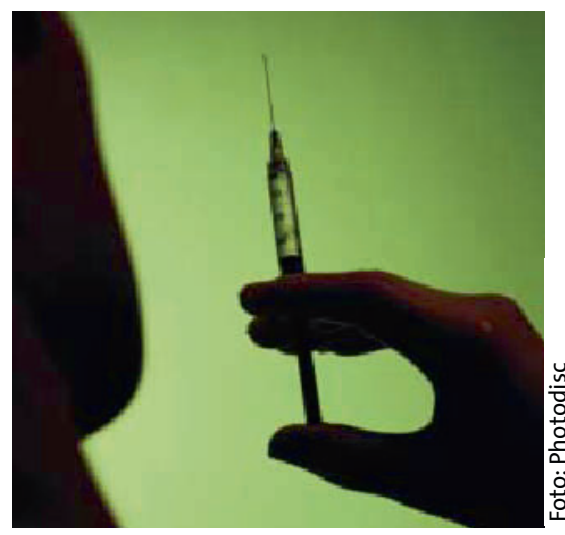

In den USA kommt es zu einem tödlichen Zwischenfall auf eine Million Injektionen im Rahmen einer SIT. der Patient an Asthma - in einer von derselben Arbeitsgruppe bereits publizierten Analyse von tödlichen Zwischenfällen traf dies bei $88 \%$ der Fälle zu. Bei $80 \%$ der Patienten trat eine Hypotonie auf, eine Ateminsuffizienz bei 10\%. Hautreaktionen fehlten bei 30\% der Patienten. Bei $6 \%$ der fast tödlichen Zwischenfälle wurde Adrenalin überhaupt nicht bzw. verspätet gegeben - bei den tödlichen Ereignissen war dies bei 30\% der Fall gewesen. Als wichtigste Ursachen für einen Zwischenfall gaben die Ärzte eine starke Allergenbelastung im Therapiezeitraum (46\%) und Dosierungsfehler $(25 \%)$ an.

Fazit: Im Rahmen einer SIT sind gesicherte fast tödliche Zwischenfälle dieser retrospektiven Umfrage zufolge 2,5-mal häufiger als tödliche Zwischenfälle, die Inzidenz tödlicher Zwischenfälle beträgt einer auf eine Million Injektionen. Diese US-Daten sind so nicht auf Europa zu übertragen, hier ist die Nebenwirkungsrate noch geringer.

$b k$

Amin HS et al. Evaluation of near-fatal reactions to allergen immunotherapy injections. J Allergy Clin Immunol 2006; 117: 169-75

\section{Verträglichere Schnellhyposensibilisierung}

\author{
Mit Rush-Titrationsprotokollen wird das Therapieziel der spezifischen \\ Immuntherapie (SIT) schneller erreicht, allerdings erhöht sich auch \\ die Zahl systemischer Nebenwirkungen. Kann eine Vorabmedikation \\ mit dem Anti-IgE-Antikörper Omalizumab dieses Risiko reduzieren?
}

P atienten mit einer allergischen Rhinitis aufgrund einer Sensibilisierung gegen Ragweed (Traubenkraut) erhielten randomisiert und doppelblind entweder neun Wochen lang Omalizumab (mindestens 0,016 mg pro kg Körpergewicht und $\mathrm{U} / \mathrm{ml}$ Serum-IgE im Monat) oder Plazebo, gefolgt von einer eintägigen Rush-Immuntherapie mit sechs Einzeldosen eines Ragweed-Allergenextrakts oder mit Plazebo. Danach wurden Verum- und Plazebo-Immuntherapie über zwölf Wochen fortgeführt, dazu erhielten die Probanden weiter regelmäßig entweder Omalizumab oder Plazebo injiziert.

123 Patienten beendeten alle Behandlungssitzungen. Bei den immuntherapierten Patienten stieg das spezifische IgG um den Faktor elf an, bei den mit Omalizumab behandelten Patienten sanken die freien IgE-Spiegel um den Faktor Anti-IgE- plus Immuntherapie weniger Nebenwirkungen registriert als bei Patienten unter einer alleinigen Immuntherapie. Durch die zusätzliche Gabe von Omalizumab konnte zudem das Anazehn. Dabei wurden bei Patienten unter
phylaxie-Risiko bei der Rush-Titration um das Fünffache gesenkt werden. In einer Intent-to-Treat-Auswertung zeigten die Patienten unter der Kombinationstherapie eine signifikante Verbesserung des Symptomscores während der Ragweed-Saison im Vergleich zu Patienten mit einer alleinigen SIT $(p=0,044)$.

Fazit: Bei Patienten mit einer RagweedRhinitis vermindert die Gabe von Omalizumab vor Beginn einer Rush-SIT das Risiko von systemischen Nebenwirkungen. Eine Anti-IgE-Therapie während der SIT-Erhaltungsphase könnte die $\mathrm{Hy}$ posensibilisierung noch sicherer und effektiver machen.

Casale TB et al. Omalizumab pretreatment decreases acute reactions after rush immunotherapy for ragweed-induced seasonal allergic rhinitis. J Allergy Clin Immunol 2006; 117: 134-40 